\title{
Metachronous Merkel Cell Carcinoma: A Case Report
}

\author{
Jondavid Pollock $^{\mathrm{a}}$ Thomas Caranosos $^{\mathrm{b}}$ E.P. Polack \\ a The Schiffler Cancer Center, Wheeling Hospital, Wheeling, W. Va., 'bepartment of Surgery, \\ and ${ }^{\mathrm{C} C l i n i c a l}$ Professor of Surgery, West Virginia University, Morgantown, W. Va., USA
}

\section{Key Words}

Merkel cell carcinoma $\cdot$ Metachronous lesions $\cdot$ Lymph node involvement

\begin{abstract}
Merkel cell carcinoma (MCC) typically presents as an isolated cutaneous lesion with a measurable risk of regional lymph node involvement. Uncommonly, synchronous or metachronous lesions have been described to be attributed to a field effect. This case report describes a patient presenting with metachronous lesions, separated by several years, which cannot be attributed to a field effect given the tumor distribution.
\end{abstract}

\section{Introduction}

Merkel cell carcinoma (MCC) is an uncommon cutaneous malignancy with an estimated 1,500 cases diagnosed annually in the US. It is most commonly seen in men in their 70s and on sun-exposed skin. Moreover, when it is found in immunosuppressed patients, the clinical course can be more fulminant [1]. The cell of origin remains elusive and the non-descript nature of the histology has led to various descriptions of the tumor, which include small cell cancer of the skin, trabecular cutaneous tumor, or anaplastic skin cancer [2]. Although the regional lymph nodal basin may be occultly involved in as many as $24 \%$ of cases at presentation [3], and hematogenous spread is well documented and is importantly linked to overall survival [4], simultaneous or synchronous [5] presentation of the cutaneous lesion is unusual. Even more odd than what may be explained as a regional field effect causing adjacent simultaneous lesions is the process of metachronous cutaneous lesions, particularly when the presentation is disparate in location. Herein, we present a case report of the metachronous presentation of MCC.

\section{Case Presentation}

We present the case of a male patient who first presented to medical attention in September 2005, when he was 67 years of age. He complained of a pea-sized, non-erythematous, non-pruritic lesion 
overlying the medial compartment of the left infrapatellar leg. An excisional biopsy revealed a $2.5-\mathrm{cm}$ focus of MCC with microscopic margin involvement. He was seen by one of the authors (E.P.P.) who re-excised the lesion and performed a sentinel node staging simultaneously. The re-excision revealed a remnant $1.1 \mathrm{~cm}$ of tumor with negative margins. The sentinel node procedure revealed 2 nodes involving the superficial groin, both of which contained metastatic disease. A second stage groin dissection was conducted which failed to reveal further metastatic disease. The patient underwent staging studies, including a contrast-enhanced CT scan of his chest, abdomen, and pelvis, which showed no evidence of metastatic disease. Given the regional nodal involvement and the neuroendocrine nature of the tumor, the patient was recommended adjuvant cisplatin and etoposide chemotherapy, and he completed 4 cycles. He then received complete 5,600 cGy of external electron beam teletherapy to the tumor bed plus a $2-\mathrm{cm}$ margin. His treatment course was essentially uncomplicated.

He had been followed closely on a quarterly basis with clinical examinations and annual chest radiography when, in November 2008, he developed a cystic and erythematous lesion overlying the right olecranon process ( $\underline{\text { fig. } 1}$ ).

He visited his plastic surgeon, and an excisional biopsy demonstrated a 1-cm focus of MCC with a close deep margin. He underwent a re-excision and sentinel node mapping, revealing no residual local disease and negative regional axillary nodes. A staging CT-PET fusion failed to reveal metastatic disease. Treatment with 5,000-cGy electron beam teletherapy to the tumor bed was continued, completing that course uneventfully. The patient's medical history was notable for osteoarthritis, osteoporosis, hyperlipidemia, hypertension, cardiac dysrhthymia, and vascular disease. Medications included Celebrex, Fosomax, KCl, Diovan, Toprol, Vytorin, Coumadin, Norvasc, and Tramadol. His family history was notable for a brother who died of a primary brain tumor and a daughter who had a rhabdomyosarcoma. He was a retired carpenter who consumed alcohol socially and did not use tobacco products. He denied excessive sun exposure.

\section{Discussion}

This patient example of a metachronous MCC represents a distinct entity. To our knowledge, only one previous case report of a metachronous MCC has been reported in the literature and those authors concluded that the second occurrence represented a field defect given the proximity of the two tumors as well as a sophisticated DNA comparison linking the lesions to the same cellular precursor [6]. When metachronous lesions of a rare malignancy occur, the clinician is obligated to pursue an underlying cause. There are no known risk factors associated with the development of MCC, and this malignancy is not associated with any known genetic family of cancers. A family history of a brain tumor and sarcoma suggests a potential Li-Fraumeni syndrome, though this rare mutation in the p53 gene has not been associated with MCC. Immunosuppression has been shown to increase the likelihood of unifocal MCC [7]; however, it has not been reported to increase the risk of metachronous lesions, nor does this patient have any history of conventional forms of immunosuppression. Several studies have suggested a role for polyomavirus in the etiology of MCC, and virus-specific assays assess the involvement of the infectious agent [8]. The patient presented in this report had no clinical manifestations of this virus, but a specific assay was not performed, leading us to query whether his metachronous presentation of MCC was virus-induced. Indeed, addressing the specific clonal rearrangement of the two separate lesions may have added to the evidence that the two tumors were related (perhaps in their viral association) or truly separate. Although it is tempting to identify a potential viral pathogen that may be associated with MCC, the development of an anti-viral therapy or immunization remains of scientific import only for the present and perhaps, because of the rarity of MCC, will never become a practical consideration. Because of the potential increase in risk that this patient may develop future disparate MCC lesions, regardless of the etiology, he is being 
followed closely with clinical dermatologic surveys. Other surveillance studies, such as laboratory or radiographic surveys, appear unwarranted in the absence of signs or symptoms of disease.

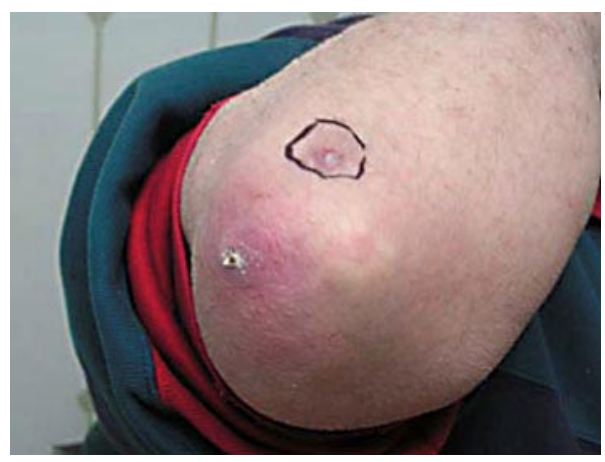

Fig. 1. Cystic and erythematous lesion overlying the right olecranon process.

\section{References}

1 Penn I, First MR: Merkel's cell carcinoma in organ recipients: report of 41 cases. Transplantation 1999;68:1717-1721.

2 Pollock J: Merkel cell carcinoma: an uncommon and challenging disease entity. Commun Oncol 2007;4:610612.

-3 Allen PJ, Browne WB, Jaques DP: Merkel cell carcinoma: prognosis and treatment of patients from a single institution. Cancer 2007;110:1-12.

-4 Poulsen MG, Rischin D, Porter I: Does chemotherapy improve survival in high-risk stage 1 and 2 Merkel cell carcinoma of the skin? Int J Radiat Oncol Biol Phys 2006;64:114-119.

-5 Satter EK, Derienzo DP: Synchronous onset of multiple cutaneous neuroendocrine (Merkel cell) carcinomas localized to the scalp. J Cutan Pathol 2008;35:685-691.

-6 Nagy J, Feher LZ, Sonkodi I, Lesznyak J, Ivanyi B, Puskas LG: A second field metachronous Merkel cell carcinoma of the lip and palantine tonsil confirmed by microarray-based comparative genomic hybridization. Virchows Arch 2005;446:278-286.

7 Lanoy E, Dores GM, Madeleine MM, Toro JR, Fraumeni JF, Engels EA: Epidemiology of nonkeratinocytic skin cancers among persons with AIDS in the United States. AIDS 2009;23:385-393.

-8 Sihto H, Kukko H, Koljonen V, Sankila R, Bohling T, Joensuu H: Merkel cell polyomavirus infection, large T antigen, retinoblastoma protein and outcome in Merkel cell carcinoma. Clin Cancer Res 2011;17:4806-4813. 